\title{
Skin-capsular Mismatch According to Knee Positions for Instrument Insertion via Arthroscopic Portals
}

\author{
Jung-Ro Yoon, MD, Venkatesh KP, MD, Taik-Sun Kim, MD, Deuk-Soo Lim, MD and Jae-Hyuk Yang, MD \\ Department of Orthopedic Surgery, Seoul Veterans Hospital, Seoul, Korea
}

Damage to soft tissues, chondral surfaces, and the menisci may result from imprise or overly aggressive establishment of portals in arthroscopic knee surgeries. In this note, we address the relationship between the skin and the capsule at portal sites according to knee positions. Understanding the skin-capsular mismatch may facilitate arthroscopic procedures and indirectly reduce the operation time.

Key words: Arthroscopy, Portals, Skin-capsular mismatch, Knee position.

Arthroscopic surgery is the most frequently performed orthopedic procedure in the world ${ }^{1,2)}$, because of its broader indications and low rate of morbidity and complications ${ }^{3}$. Easy access of instrument through the anterolateral and anteromedial portals is critical for safe and efficient knee arthroscopy.

However, in spite of refined techniques, there are possibilities for the occurrence of various complications during the orthopedic procedure. Damage to soft tissue, chondral surfaces, and the menisci may result from imprecise or overly aggressive establishment of portals. Poorly positioned portals may require creation of larger capsular incisions or additional entry sites, thus exacerbating the problem. In this note, we simply describe a relationship between the skin and joint capsule, which can be beneficial for easy instrument access.

Received April 23, 2011; Revised (1st) May 31, 2011; (2nd) July 3, 2011; Accepted July 5, 2011.

Correspondence to: Jae-Hyuk Yang, MD.

Department of Orthopedic Surgery, Seoul Veterans Hospital,

6-2 Dunchon-dong, Kangdong-gu, Seoul 134-791, Korea.

Tel: +82-2-2225-1609, Fax: +82-2-2225-1190

Email: jaekorea@gmail.com

This is an Open Access article distributed under the terms of the Creative Commons Attribution Non-Commercial License (http://creativecommons.org/licenses/by-nc/3.0/) which permits unrestricted non-commercial use, distribution, and reproduction in any medium, provided the original work is properly cited.

Copyright ( $)$ 2011. KOREAN KNEE SOCIETY

www.jksrr.org

\section{Technical Note}

The patient was placed in the supine position with the knee in a dangling position at around $70^{\circ}$ of flexion. The un-operated left leg was supported with circumferential operative leg holders with sufficient padding at the fibular head to prevent common peroneal nerve palsy caused by the pressure ${ }^{4)}$. A tourniquet was used during the surgery.

For portal placement, anatomic structures were identified. First, the patellar apex, patellar tendon and lateral femoral condyle were identified by palpation. The lateral triangle, bounded by the lateral border of the patellar tendon, the medial circumference of the lateral femoral condyle, and distally by the superior rim of the anterior horn of the lateral meniscus, was palpated.

Once the optimum site for the anterolateral arthroscopic portals are selected, a longitudinal skin incision approximately 4-5 $\mathrm{mm}$ long was made with a scalpel (No. 11). Subsequently, a vertical incision about 3-4 mm was made at the lateral capsule. An arthroscope sheath with a blunt obturator was introduced into the joint. The sheath was inserted with a careful rotatory movement, avoiding injury to the cartilage and synovial covering of the anterior cruciate ligament. When the sheath penetrated the fibrous capsule and tissue resistance declined, the knee joint was extended and the sheath was carefully advanced into the medial portion of the suprapatellar pouch.

The "needle technique" was used to make an anteromedial instrumental portal. By constantly visualizing the needle, the optimum position was decided. Next, a longitudinal skin incision was created (Fig. 1A). 
During the arthroscopic procedure, the relationship between the skin opening and capsular opening was assessed by monitoring the changes in the relative direction of the scope and the instruments. In an extended knee position $\left(20^{\circ}\right)$, the capsular opening migrated proximally and the direction from the skin to the capsule became cephalad (Fig. 1B). In a more flexed knee position, the capsular opening migrated distally and the direction from the skin to the capsule moved caudally (Fig. 1C). In the valgus and varus stressed knee position, the capsular opening migrated slightly laterally and medially, respectively. The commonly used position in knee arthroscopy is valgus extended knee for the examination of the medial meniscus and varus flexed knee (figure of " 4 " position) for the lateral meniscus. At $30^{\circ}$ of knee flexion with valgus stress, the direction from the skin to the capsule changed cephalad with a slight lateral tilt. At $90^{\circ}$ of knee flexion with varus stress, the direction from the skin to the capsule changed to a caudal with a slight medial tilt. To confirm the mismatch between the skin and the capsule according to various knee positions, capsular suture was carried out using roll wire (No. 2, Zimmer, Warsaw, IN, USA). The skin was temporarily sutured with staples (Skin Stapler, dimensions 6.9×3.9 mm, Johnson \& Johnson Company, Cincinnati, OH, USA). The relative positions were confirmed by intraoperative real-time C-arm images in various knee position, as described (Figs. 2, 3).

By knowing the direction of portals according to the knee positions, it was possible to access the portals with instruments without changing to the original dangling position or further violating the capsule.

\section{Discussion}

Even for experienced surgeons, changing the arthroscopic instruments through the knee portals can be a cumbersome process. This is due to the mismatch of the initial relationships between the skin and soft tissue orifices according to the knee position. In these situations, changing the knee position into the original (index) position or further spreading the portal opening using a mosquito may lead to prolonged operation time and
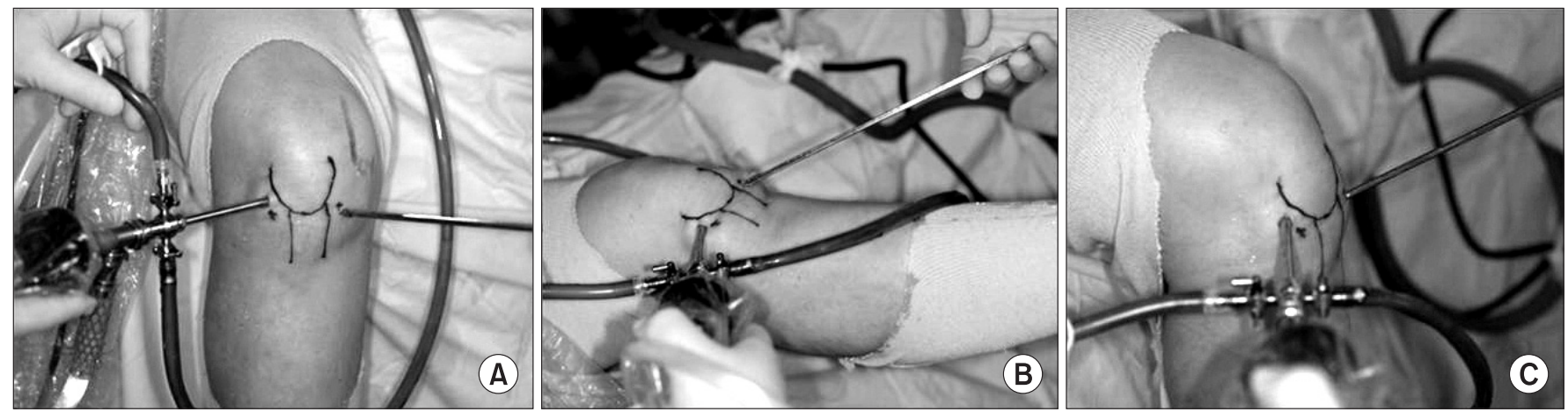

Fig. 1. (A) Right knee in dangling position, a picture showing that the direction from the skin to the capsule is right angled to the skin surface. (B) At $20^{\circ}$ of knee flexion, the direction from the skin to the capsule became cephalad. (C) At $90^{\circ}$ of knee flexion, the direction from the skin to the capsule moved caudally.
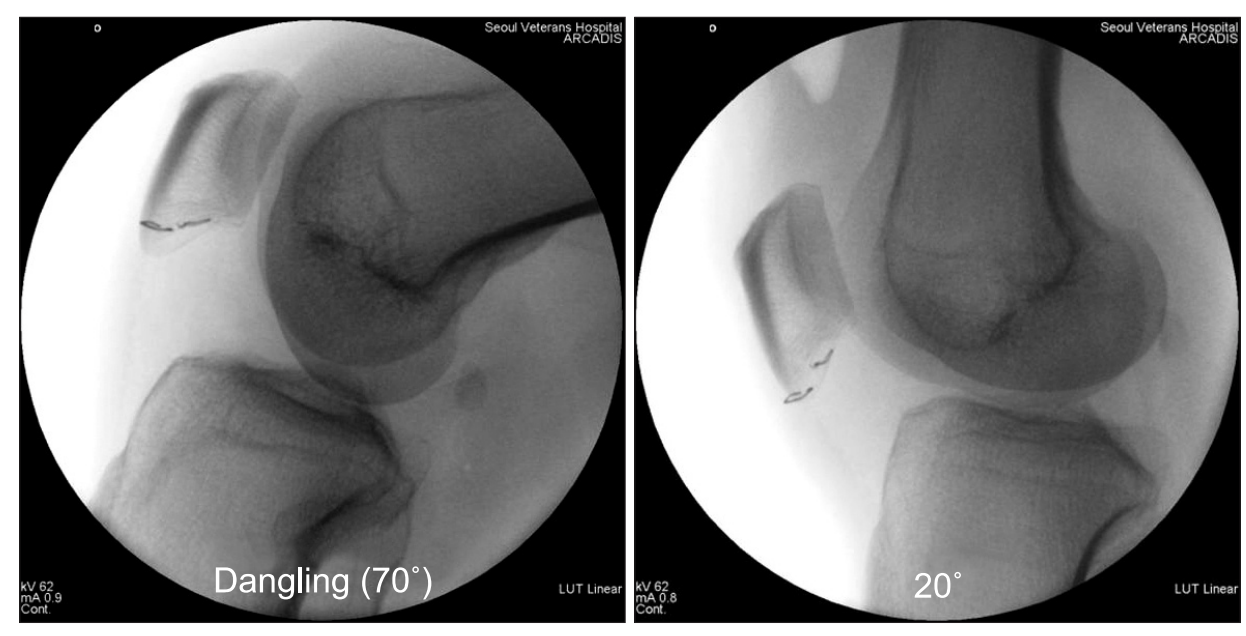

Fig. 2. Intraoperative C-arm images demonstrating the relative positions of roll wire (sutured to capsule) and the staple (sutured to skin). Notice that the roll wire migrates relatively proximally in knee extension position $\left(20^{\circ}\right)$. 

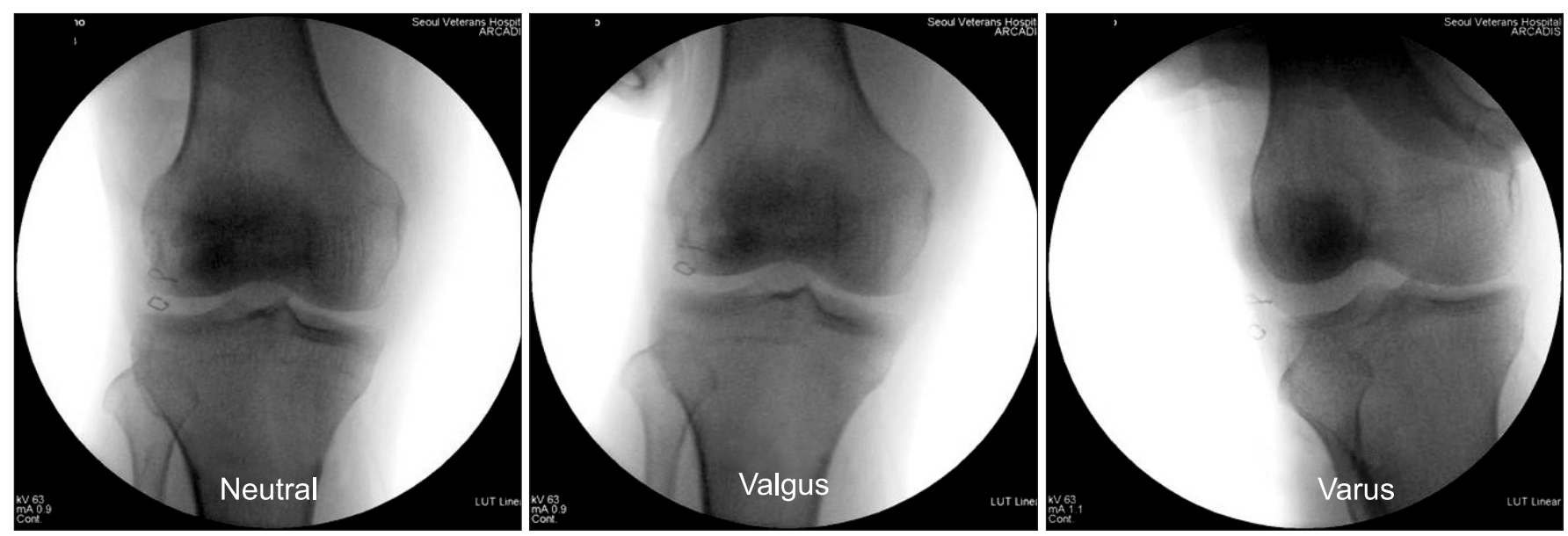

Fig. 3. Intraoperative C-arm images demonstrating the relative positions of roll wire (sutured to capsule) and the staple (sutured to skin). Relative positions are shown in valgus and varus stress (figure of "4") position.

further violation of the capsule. By understanding the relative skin-capsular mismatch in arthroscopic portals according to knee positions, instrument insertion can be facilitated with the adjustment of the direction of instrument insertion or skin traction.

We believe that knowing the direction of portals according to knee position is useful during arthroscopic procedures. The small size of the capsular incision coupled with the elimination of frequent capsular violation limits fluid extravasation, which can complicate a straightforward procedure and lead to increased postoperative swelling. In this series, it was accessible to all portals without any difficulty.

The key point in arthroscopic procedure is "triangulation". However, a high diagnostic accuracy can only be achieved through experience ${ }^{5}$. Various authors have reported new techniques and approaches for easy access of the arthroscopic instruments into the different regions of the knee compartments with their own limitations. Kim and $\mathrm{Kim}^{6}$ ) reported different locations of portals to visualize the different regions of the compartments and concluded that the locations should be flexible as per the need of the surgeon rather than fixed. Ahn and $\mathrm{Ha}^{7)}$ showed that a posterior trans-septal portal further improves the visualization of the posterior compartments. Although different orientation of portals are available for better visualization of specific intra-articular regions, most of arthroscopic surgeries can be managed by using anterolateral, anteromedial and posteromedial portals.

This note describes the relationships between skin and soft tissue opening according to knee positions. Based on our experience of handling more than two thousand arthroscopy surgeries, we have established that correct understanding of the skin-capsular mismatch minimizes the soft tissue injury and indirectly reduces the operative time. The limitation of this study is that the degree of skin-capsular mismatch could not be shown quantitatively. Further studies with a large number of patients and an appropriate measuring method could additionally enhance the quality of the paper.

\section{References}

1. McGinty JB, Johnson LL, Jackson RW, McBryde AM, Goodfellow JW. Uses and abuses of arthroscopy: a symposium. J Bone Joint Surg Am. 1992;74:1563-77.

2. Small NC. Complications in arthroscopic surgery performed by experienced arthroscopists. Arthroscopy. 1988;4:215-21.

3. Hadied AM. An unusual complication of arthroscopy: a fistula between the knee and the prepatellar bursa. Case report. J Bone Joint Surg Am. 1984;66:624.

4. Gillquist J, Hagberg G. A new modification of the technique of arthroscopy of the knee joint. Acta Chir Scand. 1976;142: 123-30.

5. Parsons EM, Bono JV. A new technique for establishing and maintaining an anteromedial knee portal. Am J Orthop (Belle Mead NJ). 2008;37:323-4.

6. Kim SJ, Kim HJ. High portal: Practical philosophy for positioning portals in knee arthroscopy. Arthroscopy. 2001;17:333-7.

7. Ahn JH, Ha CW. Posterior trans-septal portal for arthroscopic surgery of the knee joint. Arthroscopy. 2000;16:774-9. 